TRANSACTIONS OF THE

AMERICAN MATHEMATICAL SOCIETY

Volume 354, Number 6, Pages 2187-2205

S 0002-9947(02)02964-1

Article electronically published on February 14, 2002

\title{
LOCAL SUBGROUPS AND THE STABLE CATEGORY
}

\author{
WAYNE W. WHEELER
}

\begin{abstract}
If $G$ is a finite group and $k$ is an algebraically closed field of characteristic $p>0$, then this paper uses the local subgroup structure of $G$ to define a category $\mathfrak{L}(G, k)$ that is equivalent to the stable category of all left $k G$-modules modulo projectives. A subcategory of $\mathfrak{L}(G, k)$ equivalent to the stable category of finitely generated $k G$-modules is also identified. The definition of $\mathfrak{L}(G, k)$ depends largely but not exclusively upon local data; one condition on the objects involves compatibility with respect to conjugations by arbitrary group elements rather than just elements of $p$-local subgroups.
\end{abstract}

\section{INTRODUCTION}

One of the main themes of modular representation theory, going back many decades to the fundamental work of $\mathrm{R}$. Brauer, is the idea that the representations of a finite group are closely related to those of its local subgroups. To some extent this paper is intended to provide a general explanation for why this idea has proven to be so fruitful over such a long period. In particular, the work presented here shows that it is possible to use the local subgroups of a finite group to construct a category equivalent to the stable category.

Let $G$ be a finite group, let $k$ be an algebraically closed field of characteristic $p$, and let $\mathcal{P}(G)$ be the collection of all $p$-subgroups of $G$. The stable category $k G$-Mod is obtained by factoring out the projective modules from the category of all left $k G$ modules. Section 3 defines a category $\mathfrak{L}(G, k)$ in which the objects are essentially certain collections of modules. In particular, an object $L$ in $\mathfrak{L}(G, k)$ determines a module $L(P)$ in $k N_{G}(P)$-Mod for each $P \in \mathcal{P}(G)$. Each module $L(P)$ must satisfy a condition on its variety, and the family of all modules determined by $L$ must be compatible under conjugation and restriction. In the compatibility conditions conjugations by arbitrary group elements are allowed, so the definition of $\mathfrak{L}(G, k)$ does not depend solely on the local structure of $G$. The main result of this paper is that $\mathfrak{L}(G, k)$ is equivalent to $k G$-Mod. If $Q$ is a Sylow $p$-subgroup of $G$, let $\mathfrak{l}(G, k)$ be the full subcategory of $\mathfrak{L}(G, k)$ consisting of the objects $L$ such that $L(Q)$ is stably isomorphic to a finitely generated module. Then $\mathfrak{l}(G, k)$ is equivalent to the full subcategory $k G$-mod of finitely generated modules in $k G$-Mod.

The work presented here makes extensive use of Rickard's work on idempotent modules 3 as well as Benson, Carlson, and Rickard's theory of varieties for infinitely generated modules [1. These topics are reviewed in Section 2. The third section defines the category $\mathfrak{L}(G, k)$ and a canonical functor $\mathfrak{F}: k G$-Mod $\rightarrow \mathfrak{L}(G, k)$. It is possible to define the tensor product of a $k G$-module $M$ and an object $L$ of

Received by the editors January 2, 2001 and, in revised form, September 24, 2001.

2000 Mathematics Subject Classification. Primary 20C20. 
$\mathfrak{L}(G, k)$, and the result is another object of $\mathfrak{L}(G, k)$. This idea is considered in Section 4. Finally, Section 5 is devoted to proving that the canonical functor $\mathfrak{F}: k G$ - $\underline{\operatorname{Mod}} \rightarrow \mathfrak{L}(G, k)$ is an equivalence of categories.

\section{Preliminary Results}

Throughout this paper $G$ denotes a finite group, and $k$ is an algebraically closed field of prime characteristic $p$. If $g \in G$ and $H$ is a subgroup of $G$, let ${ }^{g} H=g H^{-1}$. For any $k H$-module $M$ set $M \uparrow^{G}=k G \otimes_{k H} M$; if $M$ is a $k G$-module, then $M \downarrow_{H}$ denotes the restriction of $M$ to $H$. It will often be convenient simply to write $M \downarrow$ when the subgroup $H$ can be inferred from the context. We write $\varepsilon: k_{H}^{\uparrow G} \rightarrow k$ for the augmentation map given by

$$
\varepsilon\left(\sum_{g \in G / H} g \otimes x_{g}\right)=\sum_{g} x_{g} .
$$

Let $k G$-Mod denote the category of all left $k G$-modules, and let $k G$-mod be the full subcategory of finitely generated $k G$-modules. If $M$ and $M^{\prime}$ are $k G$-modules, let $\operatorname{PHom}_{k G}\left(M, M^{\prime}\right)$ denote the $k$-subspace of $\operatorname{Hom}_{k G}\left(M, M^{\prime}\right)$ consisting of those maps that factor through a projective $k G$-module. The stable category $k G$-Mod has the same objects as $k G$-Mod, but the morphisms from $M$ to $M^{\prime}$ in $k G$ - $\underline{\mathrm{Mod}}$ are defined by setting

$$
\underline{\operatorname{Hom}}_{k G}\left(M, M^{\prime}\right)=\operatorname{Hom}_{k G}\left(M, M^{\prime}\right) / \operatorname{PHom}_{k G}\left(M, M^{\prime}\right) \text {. }
$$

The full subcategory of $k G$-Mod consisting of finitely generated $k G$-modules is denoted $k G$-mod. It is well known that the categories $k G$-Mod and $k G$-mod are triangulated (Theorem I.2.6 of [2]), and the translation functor is given by $\Omega^{-1}$. For convenience we often identify $\Omega^{-1}$ with the isomorphic functor $\Omega^{-1} k \otimes-$.

If $\gamma: M \rightarrow M^{\prime}$ is a $k G$-homomorphism, then we normally also write $\gamma$ for the corresponding map in $k G$-Mod. In fact, we will generally only be concerned with maps in the stable category. In a few cases homomorphisms are defined in the module category, but even then it is always the image in $k G$-Mod that is of interest.

Recall that if $\mathcal{T}$ is a triangulated category and $\mathcal{C}$ is a full triangulated subcategory of $\mathcal{T}$, then $\mathcal{C}$ is said to be a thick subcategory if it is closed under taking direct summands of objects. Now suppose that $\mathcal{C}$ is a thick subcategory of $k G-\underline{\bmod }$. As in [3], we say that $\mathcal{C}$ is a tensor-ideal subcategory of $k G$-mod if $M \otimes M^{\prime}$ is in $\mathcal{C}$ whenever $M$ is in $\mathcal{C}$ and $M^{\prime}$ is in $k G$-mod. Let $\mathcal{C}^{\oplus}$ denote the smallest full triangulated subcategory of $k G$-Mod that contains $\mathcal{C}$ and is closed under arbitrary direct sums. A module $M$ is said to be $\mathcal{C}$-local if $\underline{\operatorname{Hom}}_{k G}(C, M)=0$ for all $C$ in $\mathcal{C}$.

The following proposition summarizes the fundamental facts about idempotent modules that will be needed in the following sections. Proofs can be found in $\underline{3}$.

Proposition 2.1. Let $\mathcal{C}$ be a tensor-ideal subcategory of $k G$-mod. For any object $M$ in $k G$-Mod there is a triangle

$$
e_{\mathcal{C}}(M) \longrightarrow M \longrightarrow f_{\mathcal{C}}(M) \longrightarrow \Omega^{-1}\left(e_{\mathcal{C}}(M)\right)
$$

in $k G$-Mod such that $e_{\mathcal{C}}(M)$ is in $\mathcal{C}^{\oplus}$ and $f_{\mathcal{C}}(M)$ is $\mathcal{C}$-local, and such a triangle is unique up to isomorphism. The morphism $e_{\mathcal{C}}(M) \rightarrow M$ is the universal map in $k G$-Mod from an object of $\mathcal{C}^{\oplus}$ to $M$, and $M \rightarrow f_{\mathcal{C}}(M)$ is the universal map from $M$ to a $\mathcal{C}$-local object. The modules $e_{\mathcal{C}}(k)$ and $f_{\mathcal{C}}(k)$ are idempotent in the 
sense that $e_{\mathcal{C}}(k) \otimes e_{\mathcal{C}}(k) \cong e_{\mathcal{C}}(k)$ and $f_{\mathcal{C}}(k) \otimes f_{\mathcal{C}}(k) \cong f_{\mathcal{C}}(k)$ in $k G$-Mod. Moreover, $e_{\mathcal{C}}(k) \otimes M \cong e_{\mathcal{C}}(M)$ and $f_{\mathcal{C}}(k) \otimes M \cong f_{\mathcal{C}}(M)$ in $k G$-Mod for any module $M$.

In the following work it will often be necessary to consider the universal map $\eta: e_{\mathcal{C}}(k) \rightarrow k$ described in Proposition 2.1. By abuse of notation we usually use the same symbol $\eta$ to denote this map for any thick subcategory $\mathcal{C}$ of $k G$-mod and for any finite group $G$.

Benson, Carlson, and Rickard have used idempotent modules to develop a theory of varieties for arbitrary $k G$-modules, and we give a brief review of this theory. Although it is common to consider the maximal ideal spectrum of the cohomology ring $H^{*}(G, k)$, we will use the space $\operatorname{Proj} H^{*}(G, k)$ of all homogeneous prime ideals that do not contain the ideal $\bigoplus_{n=1}^{\infty} H^{n}(G, k)$. If $I$ is a homogeneous ideal in $H^{*}(G, k)$, we set $\bar{V}_{G}(I)=\left\{\mathfrak{p} \in \operatorname{Proj} H^{*}(G, k) \mid I \subseteq \mathfrak{p}\right\}$. If $M$ is a finitely generated $k G$-module, let $J(M)$ be the annihilator of $\operatorname{Ext}_{k G}^{*}(M, M)$ in $H^{*}(G, k)$, and let $\bar{V}_{G}(M)=\bar{V}_{G}(J(M))$. Then $\bar{V}_{G}(M)$ is closed in $\operatorname{Proj} H^{*}(G, k)$, and $\bar{V}_{G}(k)=\operatorname{Proj} H^{*}(G, k)$.

If $M$ is infinitely generated, however, then the definition of $\bar{V}_{G}(M)$ is more complicated. For $\mathfrak{p} \in \bar{V}_{G}(k)$ let $\mathcal{C}(\mathfrak{p})$ denote the full subcategory of $k G$-mod consisting of all finitely generated $k G$-modules $M^{\prime}$ such that $\bar{V}_{G}\left(M^{\prime}\right) \subseteq \bar{V}_{G}(\mathfrak{p})$, and let $\mathcal{C}^{\prime}(\mathfrak{p})$ denote the full subcategory of $\mathcal{C}(\mathfrak{p})$ consisting of all finitely generated modules $M^{\prime}$ such that $\mathfrak{p} \notin \bar{V}_{G}\left(M^{\prime}\right)$. Then $\mathcal{C}(\mathfrak{p})$ and $\mathcal{C}^{\prime}(\mathfrak{p})$ are tensor-ideal subcategories of $k G$ mod. If $M$ is an arbitrary $k G$-module, then $\bar{V}_{G}(M)$ can be defined as the collection of all primes $\mathfrak{p} \in \bar{V}_{G}(k)$ such that $f_{\mathcal{C}^{\prime}(\mathfrak{p})} \otimes e_{\mathcal{C}(\mathfrak{p})} \otimes M$ is not projective.

Although the set $\bar{V}_{G}(M)$ is not necessarily closed in $\bar{V}_{G}(k)$ if $M$ is not finitely generated, these sets do retain the most important properties of varieties for finitely generated modules. In particular, the following results hold for any $k G$-modules $M$ and $M^{\prime}$ :

(1) $M$ is projective if and only if $\bar{V}_{G}(M)=\emptyset$;

(2) $\bar{V}_{G}\left(M \oplus M^{\prime}\right)=\bar{V}_{G}(M) \cup \bar{V}_{G}\left(M^{\prime}\right)$;

(3) $\bar{V}_{G}\left(M \otimes M^{\prime}\right)=\bar{V}_{G}(M) \cap \bar{V}_{G}\left(M^{\prime}\right)$.

The reader should be warned, however, that the definition of $\bar{V}_{G}(M)$ given here is not used in [1]. In that paper the authors define the so-called variety $\mathcal{V}_{G}(M)$ of a module $M$ to be a collection of homogeneous irreducible subvarieties of the maximal ideal spectrum of $H^{*}(G, k)$. The results of [1] can be translated into the notation used here by observing that $V \in \mathcal{V}_{G}(M)$ if and only if the generic point of $V$ lies in $\bar{V}_{G}(M)$.

Now let $V$ be a closed subset of $\bar{V}_{G}(k)$, and let $\mathcal{C}(V)$ denote the full subcategory of $k G$-mod consisting of all finitely generated $k G$-modules $M$ such that $\bar{V}_{G}(M) \subseteq V$. For simplicity we write $e_{V}$ for $e_{\mathcal{C}(V)}$ and $f_{V}$ for $f_{\mathcal{C}(V)}$. If $H$ is a subgroup of $G$, let $\operatorname{res}_{G, H}^{*}: \bar{V}_{H}(k) \rightarrow \bar{V}_{G}(k)$ denote the map induced by the restriction homomorphism $\operatorname{res}_{G, H}: H^{*}(G, k) \rightarrow H^{*}(H, k)$.

Proposition 2.2. Let $V$ and $W$ be closed subsets of $\bar{V}_{G}(k)$, and let $H$ be a subgroup of $G$. Then

(1) $e_{V} \otimes e_{W} \cong e_{V \cap W}$ and $f_{V} \otimes f_{W} \cong f_{V \cup W}$ in $k G$-Mod;

(2) $e_{V} \downarrow_{H} \cong e_{\left(\operatorname{res}_{G, H}^{*}\right)^{-1}(V)}$ and $f_{V} \downarrow_{H} \cong f_{\left(\operatorname{res}_{G, H}^{*}\right)^{-1}(V)}$;

(3) $\bar{V}_{G}\left(e_{V}\right)=V$ and $\bar{V}_{G}\left(f_{V}\right)=\bar{V}_{G}(k)-V$.

Proof. The first two statements are proven in [3]; the third statement is Proposition 3.1 of [4]. 
Proposition 2.3. Suppose that $H$ is a subgroup of $G$.

(1) If $M$ is a $k G$-module, then $\bar{V}_{H}\left(M \downarrow_{H}\right)=\left(\operatorname{res}_{G, H}^{*}\right)^{-1}\left(\bar{V}_{G}(M)\right)$.

(2) If $M$ is a $k H$-module, then $\bar{V}_{G}\left(M \uparrow^{G}\right)=\operatorname{res}_{G, H}^{*}\left(\bar{V}_{H}(M)\right)$.

Proof. See Propositions 4.1 and 4.2 of [4].

Now suppose that $P$ is an arbitrary $p$-subgroup of $G$, and define

$$
V_{G, P}=\operatorname{res}_{G, P}^{*}\left(\bar{V}_{P}(k)\right) .
$$

We shall write $e_{G, P}$ for $e_{V_{G, P}}$ and $f_{G, P}$ for $f_{V_{G, P}}$. If $E$ is an elementary abelian $p$-subgroup of $G$, set

$$
V_{G, E}^{-}=\bigcup_{E_{0}<E} V_{G, E_{0}}
$$

and $V_{G, E}^{+}=V_{G, E}-V_{G, E}^{-}$. Then the module $\lambda_{G, E}=f_{V_{G, E}^{-}} \otimes e_{V_{G, E}}$ satisfies $\bar{V}_{G}\left(\lambda_{G, E}\right)=V_{G, E}^{+}$.

Proposition 2.4. Let $E$ be an elementary abelian p-subgroup of $G$, and set $N=$ $N_{G}(E)$. Then $\lambda_{N, E}^{\uparrow G}$ is stably isomorphic to $\lambda_{G, E}$. Moreover, the map $1 \otimes \varepsilon \eta \uparrow^{G}$ : $\lambda_{G, E} \otimes e_{N, E}^{\uparrow G} \rightarrow \lambda_{G, E}$ is a stable isomorphism.

Proof. The first statement is Proposition 4.5 of [4]. The proof of that proposition also shows that the second statement is true, although it is not explicitly stated in [4.

Lemma 2.5. Let $V, W$, and $X$ be closed subsets of $\bar{V}_{G}(k)$, and assume that $W \cap$ $X \subseteq V$. Then there are canonical stable isomorphisms $\left(f_{V} \otimes e_{W}\right) \oplus\left(f_{V} \otimes e_{X}\right) \rightarrow$ $f_{V} \otimes e_{W \cup X}$ and $f_{V} \otimes e_{W} \rightarrow f_{V \cup X} \otimes e_{W}$.

Proof. See Lemma 3.2 of [4].

Let $r$ be the $p$-rank of $G$. For $0 \leq s \leq r$ let

$$
V_{s}=\bigcup_{E} V_{G, E},
$$

where the union is taken over all elementary abelian $p$-subgroups $E$ of $G$ with $\operatorname{rank} E \leq s$. Set $e_{s}=e_{V_{s}}$ and $f_{s}=f_{V_{s}}$.

The proof of the following result is similar to that of Proposition 4.3 of [4].

Proposition 2.6. Let $0 \leq s \leq r-1$, and let $E_{1}, \ldots, E_{n}$ be a set of representatives for the conjugacy classes of elementary abelian p-subgroups of rank $s+1$ in $G$. Then

$$
f_{s} \otimes e_{s+1} \cong \bigoplus_{i=1}^{n} \lambda_{G, E_{i}} .
$$

Proof. For $1 \leq i \leq n$ set $V_{s+1}^{(i)}=V_{G, E_{i}}$, and let $V_{s+1}^{(n+1)}$ be the union of all components of $V_{s+1}$ of dimension at most $s$. Then $V_{s+1}=V_{s+1}^{(1)} \cup \cdots \cup V_{s+1}^{(n+1)}$, and we prove by induction on $t$ that $f_{s} \otimes e_{V_{s+1}^{(1)} \cup \cdots \cup V_{s+1}^{(t)}} \cong \bigoplus_{i=1}^{t} f_{s} \otimes e_{V_{s+1}^{(i)}}$ for $1 \leq t \leq n+1$. There is nothing to prove if $t=1$, so assume that $1<t \leq n+1$. Because $\left(V_{s+1}^{(1)} \cup \cdots \cup V_{s+1}^{(t-1)}\right) \cap V_{s+1}^{(t)} \subseteq V_{s}$, Lemma2.5 and the inductive assumption give

$$
f_{s} \otimes e_{V_{s+1}^{(1)} \cup \cdots \cup V_{s+1}^{(t)}} \cong\left(f_{s} \otimes e_{V_{s+1}^{(1)} \cup \cdots \cup V_{s+1}^{(t-1)}}\right) \oplus\left(f_{s} \otimes e_{V_{s+1}^{(t)}}\right) \cong \bigoplus_{i=1}^{t} f_{s} \otimes e_{V_{s+1}^{(i)}},
$$


as desired. Moreover, $V_{s+1}^{(n+1)} \subseteq V_{s}$, so that $f_{s} \otimes e_{V_{s+1}^{(n+1)}} \cong 0$ in $k G$-Mod. Hence

$$
f_{s} \otimes e_{s+1} \cong \bigoplus_{i=1}^{n+1} f_{s} \otimes e_{V_{s+1}^{(i)}} \cong \bigoplus_{i=1}^{n} f_{s} \otimes e_{V_{G, E_{i}}}
$$

Fix $i$ with $1 \leq i \leq n$, and let $V_{i}^{\prime}$ be the union of all components of $V_{s}$ that are not contained in $V_{G, E_{i}}^{-}$. Then $V_{G, E_{i}} \cap V_{i}^{\prime} \subseteq V_{G, E_{i}}^{-}$, so Lemma 2.5] implies that

$$
f_{s} \otimes e_{V_{G, E_{i}}}=f_{V_{G, E_{i}}^{-} \cup V_{i}^{\prime}} \otimes e_{V_{G, E_{i}}} \cong f_{V_{G, E_{i}}^{-}} \otimes e_{V_{G, E_{i}}}=\lambda_{G, E_{i}} \text {. }
$$

Thus $f_{s} \otimes e_{s+1} \cong \bigoplus_{i=1}^{n} \lambda_{G, E_{i}}$, as desired.

Proposition 2.7. Let $0 \leq s \leq r-1$, and let $E_{1}, \ldots, E_{n}$ be a set of representatives for the conjugacy classes of elementary abelian p-subgroups of rank $s+1$ in $G$. Set $N_{j}=N_{G}\left(E_{j}\right)$ for all $j$, and let $\eta_{j}: e_{N_{j}, E_{j}} \rightarrow k$ be the universal map described in Proposition 2.1. Then for $1 \leq j \leq n$ there are maps $\alpha_{j}: e_{N_{j}, E_{j}}^{\uparrow G} \rightarrow e_{s+1}$ such that $\eta \alpha_{j}=\varepsilon \eta_{j} \uparrow^{G}$ and $1 \otimes\left(\bigoplus_{j=1}^{n} \alpha_{j}\right): f_{s} \otimes\left(\bigoplus_{j=1}^{n} e_{N_{j}, E_{j}}^{\uparrow G}\right) \rightarrow f_{s} \otimes e_{s+1}$ is a stable isomorphism.

Proof. For each $j$ there is a commutative diagram

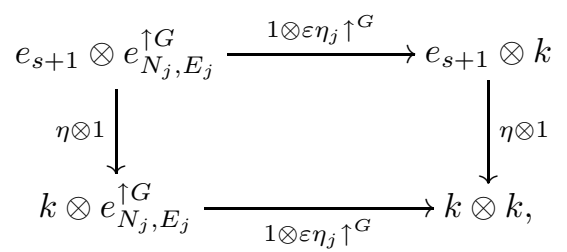

and $\bar{V}_{G}\left(e_{N_{j}, E_{j}}^{\uparrow G}\right)=V_{G, E_{j}} \subseteq V_{s+1}$, so that $\eta \otimes 1: e_{s+1} \otimes e_{N_{j}, E_{j}}^{\uparrow G} \rightarrow e_{N_{j}, E_{j}}^{\uparrow G}$ is a stable isomorphism for all $j$. Set $\alpha_{j}=\left(1 \otimes \varepsilon \eta_{j} \uparrow^{G}\right)(\eta \otimes 1)^{-1}$. Then the above diagram shows that $\eta \alpha_{j}=\varepsilon \eta_{j} \uparrow^{G}$ for all $j$.

To prove that $1 \otimes\left(\bigoplus_{j=1}^{n} \alpha_{j}\right): f_{s} \otimes\left(\bigoplus_{j=1}^{n} e_{N_{j}, E_{j}}^{\uparrow G} \rightarrow f_{s} \otimes e_{s+1}\right.$ is a stable isomorphism, it suffices to prove that $1 \otimes 1 \otimes\left(\bigoplus_{j=1}^{n} \varepsilon \eta_{j} \uparrow^{G}\right): f_{s} \otimes e_{s+1} \otimes$ $\left(\bigoplus_{j=1}^{n} e_{N_{j}, E_{j}}^{\uparrow G}\right) \rightarrow f_{s} \otimes e_{s+1}$ is a stable isomorphism. Now $f_{s} \otimes e_{s+1} \cong \bigoplus_{i=1}^{n} \lambda_{G, E_{i}}$, and $\bar{V}_{G}\left(\lambda_{G, E_{i}} \otimes e_{N_{j}, E_{j}}^{\uparrow G}\right)=V_{G, E_{i}}^{+} \cap V_{G, E_{j}}=\emptyset$ if $i \neq j$. Thus it is only necessary to show that $\bigoplus_{j=1}^{n}\left(1 \otimes \varepsilon \eta_{j} \uparrow^{G}\right): \bigoplus_{j=1}^{n}\left(\lambda_{G, E_{j}} \otimes e_{N_{j}, E_{j}}^{\uparrow G}\right) \rightarrow \bigoplus_{j=1}^{n} \lambda_{G, E_{j}}$ is a stable isomorphism. But this is an immediate consequence of Proposition 2.4

\section{THE CATEGORY OF LOCAL MODULES}

The main purpose of this section is to define the category $\mathfrak{L}(G, k)$ of $G$-local modules and a canonical functor $\mathfrak{F}: k G$-Mod $\rightarrow \mathfrak{L}(G, k)$ that will be studied in the following sections. It will be useful to begin by fixing some notation. Let $\mathcal{P}(G)$ be the collection of all $p$-subgroups of $G$. If $P \in \mathcal{P}(G)$, then throughout the remainder of the paper we usually write $N$ for $N_{G}(P)$. Similar notation will be used for normalizers of other $p$-subgroups. For example, if $P_{0}, P_{1} \in \mathcal{P}(G)$, then we write $N_{0}$ for $N_{G}\left(P_{0}\right)$ and $N_{1}$ for $N_{G}\left(P_{1}\right)$.

Suppose that for every $P \in \mathcal{P}(G)$ we have a module $L(P)$ in $k N$-Mod such that $\bar{V}_{N}(L(P)) \subseteq V_{N, P}$. Assume in addition that whenever $P_{1} \subseteq P_{2}$ in $\overline{\mathcal{P}(G)}$, there is a 
homomorphism $\phi_{P_{1}, P_{2}}: L\left(P_{1}\right) \downarrow_{N_{1} \cap N_{2}} \rightarrow L\left(P_{2}\right) \downarrow_{N_{1} \cap N_{2}}$ such that the diagram

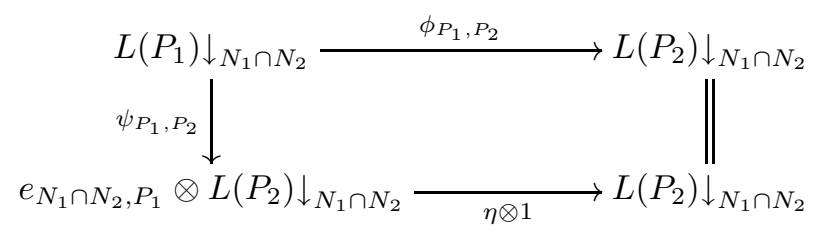

commutes for some stable isomorphism $\psi_{P_{1}, P_{2}}$. Assume that $\phi_{P, P}=1_{L(P)}$ for all $P \in \mathcal{P}(G)$ and that $\phi_{P_{2}, P_{3}} \circ \phi_{P_{1}, P_{2}}=\phi_{P_{1}, P_{3}}$ in $k\left[N_{1} \cap N_{2} \cap N_{3}\right]$-Mod whenever $P_{1} \subseteq P_{2} \subseteq P_{3}$ in $\mathcal{P}(G)$. For each $g \in G$ assume that there is a stable isomorphism $c_{g}(P): g \otimes L(P) \rightarrow L\left({ }^{g} P\right)$ such that the diagram

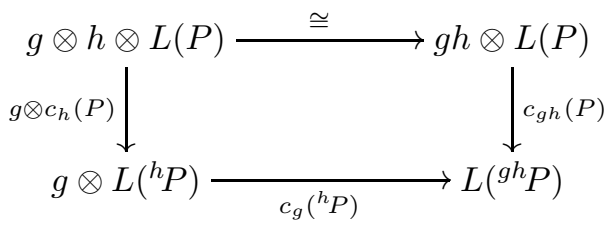

commutes for all $g, h \in G$ and all $P \in \mathcal{P}(G)$; assume in addition that if $g \in N$, then $c_{g}(P): g \otimes L(P) \rightarrow L(P)$ is the map given by $g \otimes x \mapsto g x$. Finally, suppose that if $P_{1} \subseteq P_{2}$ and $g \in G$, then there is a commutative diagram

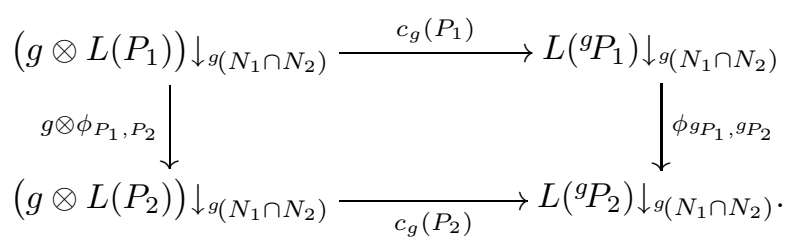

Then we say that $(L, \phi, c)$ is a $G$-local module (over $k$ ). We usually abbreviate the notation by writing $L$ for the $G$-local module $(L, \phi, c)$. For simplicity we sometimes also write $\phi$ instead of $\phi_{P_{1}, P_{2}}$ when the subgroups $P_{1}$ and $P_{2}$ can be determined from the context.

If $L=(L, \phi, c)$ and $L^{\prime}=\left(L^{\prime}, \phi^{\prime}, c^{\prime}\right)$ are $G$-local modules, let $\xi(P): L(P) \rightarrow L^{\prime}(P)$ be a map in $k N$-Mod for all $P \in \mathcal{P}(G)$. Assume that there is a commutative diagram

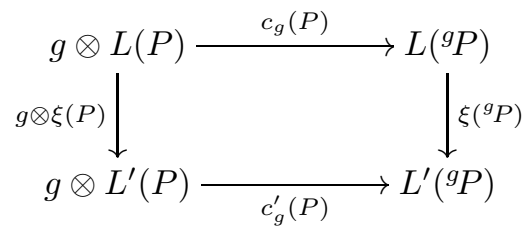

for all $P \in \mathcal{P}(G)$ and $g \in G$; assume in addition that if $P_{1}, P_{2} \in \mathcal{P}(G)$ and $P_{1} \subseteq P_{2}$, then there is a commutative diagram

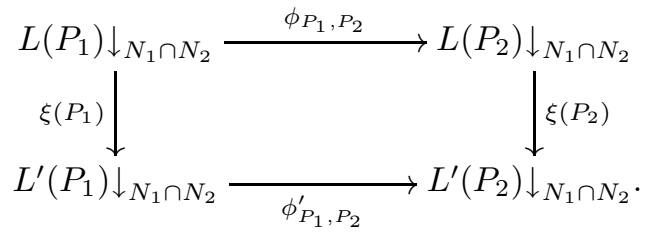


Under these circumstances we say that the sequence of maps $\xi=\{\xi(P)\}_{P \in \mathcal{P}(G)}$ is a $G$-local homomorphism.

Let $\mathfrak{L}(G, k)$ denote the category in which the objects are the $G$-local modules and the morphisms are the $G$-local homomorphisms. Our next objective is to define a canonical functor $\mathfrak{F}: k G$-Mod $\rightarrow \mathfrak{L}(G, k)$. For any $k G$-module $M$ set $(\mathfrak{F} M)(P)=$ $e_{N, P} \otimes M \downarrow_{N}$ for all $P \in \mathcal{P}(G)$. The map $\phi_{P_{1}, P_{2}}:(\mathfrak{F} M)\left(P_{1}\right) \rightarrow(\mathfrak{F} M)\left(P_{2}\right)$ is defined to be the composition

$$
\begin{aligned}
& e_{N_{1}, P_{1}} \downarrow_{N_{1} \cap N_{2}} \otimes M \downarrow_{N_{1} \cap N_{2}} \stackrel{(1 \otimes \eta \otimes 1)^{-1}}{\longrightarrow} e_{N_{1}, P_{1}} \downarrow \otimes e_{N_{2}, P_{2}} \downarrow \otimes M \downarrow \\
& \stackrel{\eta \otimes 1 \otimes 1}{\longrightarrow} e_{N_{2}, P_{2}} \downarrow_{N_{1} \cap N_{2}} \otimes M \downarrow_{N_{1} \cap N_{2}}
\end{aligned}
$$

whenever $P_{1} \subseteq P_{2}$. For each $P \in \mathcal{P}(G)$ and each $g \in G$ there is a stable isomorphism

$$
g \otimes e_{N, P} \stackrel{((g \otimes 1) \otimes \eta)^{-1}}{\longrightarrow}\left(g \otimes e_{N, P}\right) \otimes e_{g_{N,},{ }_{P}} \stackrel{(g \otimes \eta) \otimes 1}{\longrightarrow} e_{g_{N}, g_{P}} .
$$

Combining this map with the isomorphism $g \otimes M \downarrow_{N} \rightarrow M \downarrow_{g_{N}}$ given by $g \otimes m \mapsto g m$, we obtain a stable isomorphism $c_{g}(P)$ by taking the composition

$$
g \otimes\left(e_{N, P} \otimes M \downarrow_{N}\right) \cong\left(g \otimes e_{N, P}\right) \otimes\left(g \otimes M \downarrow_{N}\right) \cong e_{g_{N}, g_{P}} \otimes M \downarrow_{g_{N}} .
$$

If $\gamma: M \rightarrow M^{\prime}$ is a map in $k G$-Mod, then $\mathfrak{F} \gamma$ is the $G$-local homomorphism satisfying $(\mathfrak{F} \gamma)(P)=1 \otimes \gamma: e_{N, P} \otimes M \downarrow_{N} \rightarrow e_{N, P} \otimes M^{\prime} \downarrow_{N}$ for all $P \in \mathcal{P}(G)$.

We have now defined a canonical functor $\mathfrak{F}: k G$ - $\underline{\operatorname{Mod}} \rightarrow \mathfrak{L}(G, k)$. It will sometimes be useful to consider the analogous functor $k H$-Mod $\rightarrow \mathfrak{L}(H, k)$ for some subgroup $H$ of $G$. By abuse of notation we use the same symbol $\mathfrak{F}$ to denote this functor for any subgroup of $G$.

The following proposition characterizes the isomorphisms in $\mathfrak{L}(G, k)$. The proof is straightforward and is left to the reader.

Proposition 3.1. Let $\xi: L \rightarrow L^{\prime}$ be a G-local homomorphism. Then $\xi$ is an isomorphism in $\mathfrak{L}(G, k)$ if and only if $\xi(P): L(P) \rightarrow L^{\prime}(P)$ is an isomorphism for all $P \in \mathcal{P}(G)$.

\section{TENSOR PRODUCTS}

If one thinks of a $G$-local module essentially as a $k G$-module, then one would expect to be able to define the tensor product of two $G$-local modules. Such a definition is indeed possible, but for our purposes it will be more useful to consider the tensor product of a $k G$-module and a $G$-local module. This construction has the advantage of being slightly easier to define and to use. The main result of this section is the existence of a certain natural isomorphism relating tensor products and the functor $\mathfrak{F}$. This isomorphism is needed in the next section to show that $\mathfrak{F}$ is an equivalence.

Let $M$ be a $k G$-module, and let $L=(L, \phi, c)$ be a $G$-local module. For any $P \in \mathcal{P}(G)$ set $(M \otimes L)(P)=M \downarrow_{N} \otimes L(P)$. If $P_{1}, P_{2} \in \mathcal{P}(G)$ with $P_{1} \subseteq P_{2}$, then there is a stable isomorphism $1 \otimes \phi_{P_{1}, P_{2}}: M \downarrow_{N_{1} \cap N_{2}} \otimes L\left(P_{1}\right) \downarrow_{N_{1} \cap N_{2}} \rightarrow M \downarrow_{N_{1} \cap N_{2}} \otimes$ $L\left(P_{2}\right) \downarrow_{N_{1} \cap N_{2}}$. For any $g \in G$ and $P \in \mathcal{P}(G)$ let $m_{g}(P): g \otimes M \downarrow_{N} \rightarrow M \downarrow_{g_{N}}$ be the isomorphism given by $g \otimes x \mapsto g x$. Then there is a stable isomorphism given by the composition

$$
g \otimes\left(M \downarrow_{N} \otimes L(P)\right) \cong\left(g \otimes M \downarrow_{N}\right) \otimes(g \otimes L(P)) \stackrel{m_{g}(P) \otimes c_{g}(P)}{\longrightarrow} M \downarrow_{q_{N}} \otimes L\left({ }^{g} P\right) .
$$


With these definitions it is easy to see that $M \otimes L=(M \otimes L, 1 \otimes \phi, m \otimes c)$ is again a $G$-local module. In order to provide a connection between this definition and the functor $\mathfrak{F}$, we begin with the following lemma.

Lemma 4.1. Let $L$ be a $G$-local module, and let $P_{1}, P_{2} \in \mathcal{P}(G)$. Then there is a stable isomorphism $\theta_{L}\left(P_{1}, P_{2}\right)$ of $k\left[N_{1} \cap N_{2}\right]$-modules given by the composition

$$
\begin{gathered}
e_{N_{1}, P_{1}} \downarrow_{N_{1} \cap N_{2}} \otimes L\left(P_{2}\right) \downarrow \\
\stackrel{(1 \otimes \eta \otimes \phi)^{-1}}{\cong} e_{N_{1}, P_{1}} \downarrow \otimes e_{N_{2}, P_{2}} \downarrow \otimes L\left(P_{1} \cap P_{2}\right) \downarrow \\
\stackrel{\eta \otimes 1 \otimes \phi}{\cong} e_{N_{2}, P_{2}} \downarrow \otimes L\left(P_{1}\right) \downarrow .
\end{gathered}
$$

Moreover, the isomorphism $\theta_{L}\left(P_{1}, P_{2}\right)$ is natural in $L$. For each $g \in G$ there is a commutative diagram in $\left.k^{g}\left(N_{1} \cap N_{2}\right)\right]$-Mod of the form

$$
\begin{aligned}
& g \otimes\left(e_{N_{1}, P_{1} \downarrow} \otimes L\left(P_{2}\right) \downarrow\right) \cong e_{g N_{1},{ }^{g} P_{1} \downarrow} \otimes\left(g \otimes L\left(P_{2}\right)\right) \downarrow \stackrel{1 \otimes c_{g}\left(P_{2}\right)}{\longrightarrow} e_{g_{N_{1}},{ }^{g} P_{1} \downarrow} \otimes L\left({ }^{g} P_{2}\right) \downarrow \\
& g \otimes \theta_{L}\left(P_{1}, P_{2}\right) \downarrow \downarrow \theta_{L}\left({ }^{g} P_{1},{ }^{g} P_{2}\right) \\
& g \otimes\left(e_{N_{2}, P_{2} \downarrow} \downarrow L\left(P_{1}\right) \downarrow\right) \cong e_{g_{N_{2}},{ }^{g} P_{2} \downarrow} \downarrow\left(g \otimes L\left(P_{1}\right)\right) \downarrow \underset{1 \otimes c_{g}\left(P_{1}\right)}{\longrightarrow} e_{N_{N_{2}},{ }^{g} P_{2} \downarrow} \downarrow L L\left({ }^{g} P_{1}\right) \downarrow .
\end{aligned}
$$

Furthermore, if $P_{1} \subseteq P_{2}$ and $P \in \mathcal{P}(G)$, then there is a commutative diagram in $k\left[N_{1} \cap N_{2} \cap N\right]$-Mod given by

$$
\begin{aligned}
& e_{N_{1}, P_{1}} \downarrow \otimes e_{N_{2}, P_{2} \downarrow} \unrhd L(P) \downarrow \longrightarrow e_{N_{2}, P_{2} \downarrow} \otimes e_{N_{1}, P_{1}} \downarrow \otimes L(P) \downarrow \\
& 1 \otimes \theta_{L}\left(P_{2}, P\right) \downarrow \quad \downarrow 1 \otimes \theta_{L}\left(P_{1}, P\right) \\
& e_{N_{1}, P_{1}} \downarrow \otimes e_{N, P} \downarrow \otimes L\left(P_{2}\right) \downarrow \quad e_{N_{2}, P_{2}} \downarrow \otimes e_{N, P} \downarrow \otimes L\left(P_{1}\right) \downarrow \\
& \eta \otimes 1 \otimes 1 \longrightarrow e _ { N , P \downarrow } \otimes L ( P _ { 2 } ) \downarrow \longdiv { \eta \otimes 1 \otimes \phi }
\end{aligned}
$$

Proof. Set $P_{3}=P_{1} \cap P_{2}$. Because $N_{1} \cap N_{2} \subseteq N_{3}$, we can restrict the maps $\phi_{P_{3}, P_{i}}$ to $N_{1} \cap N_{2}$ for $i=1,2$, and we claim that the resulting maps $1 \otimes \eta \otimes \phi_{P_{3}, P_{2}} \downarrow$ and $\eta \otimes 1 \otimes \phi_{P_{3}, P_{1} \downarrow}$ occurring in the definition of $\theta_{L}\left(P_{1}, P_{2}\right)$ are isomorphisms. There is a triangle of $k\left[N_{1} \cap N_{2}\right]$-modules of the form

$$
e_{N_{2} \cap N_{3}, P_{3} \downarrow} \downarrow L\left(P_{2}\right) \downarrow \stackrel{\eta \otimes 1}{\longrightarrow} L\left(P_{2}\right) \downarrow \rightarrow f_{N_{2} \cap N_{3}, P_{3}} \downarrow \otimes L\left(P_{2}\right) \downarrow \rightarrow \Omega^{-1} k \otimes e_{N_{2} \cap N_{3}, P_{3}} \downarrow \otimes L\left(P_{2}\right) \downarrow .
$$

Because $\bar{V}_{N_{1} \cap N_{2}}\left(e_{N_{1}, P_{1}} \downarrow \otimes f_{N_{2} \cap N_{3}, P_{3} \downarrow} \otimes L\left(P_{2}\right) \downarrow\right)=\emptyset$, the map $\eta \otimes 1$ occurring in this triangle becomes an isomorphism upon tensoring with $e_{N_{1}, P_{1}} \downarrow$. The commutativity of the diagram

$$
\begin{gathered}
e_{N_{1}, P_{1}} \downarrow_{N_{1} \cap N_{2}} \otimes e_{N_{2}, P_{2}} \downarrow_{N_{1} \cap N_{2}} \otimes L\left(P_{3}\right) \downarrow_{N_{1} \cap N_{2}} \stackrel{1 \otimes \eta \otimes \phi_{P_{3}, P_{2}} \downarrow}{\longrightarrow} e_{N_{1}, P_{1}} \downarrow_{N_{1} \cap N_{2}} \otimes L\left(P_{2}\right) \downarrow_{N_{1} \cap N_{2}} \\
1 \otimes 1 \otimes \psi_{P_{3}, P_{2}} \downarrow \cong
\end{gathered}
$$

shows that $1 \otimes \eta \otimes \phi_{P_{3}, P_{2}} \downarrow$ is an isomorphism. Similarly, $\eta \otimes 1 \otimes \phi_{P_{3}, P_{1} \downarrow}$ is an isomorphism, and so is $\theta_{L}\left(P_{1}, P_{2}\right)$. It is straightforward to check that this isomorphism is natural in $L$. 
Now suppose in addition that $g \in G$. Observe that there is a commutative diagram in $k\left[{ }^{g}\left(N_{1} \cap N_{2}\right)\right]$ - $\underline{\text { Mod }}$ of the form

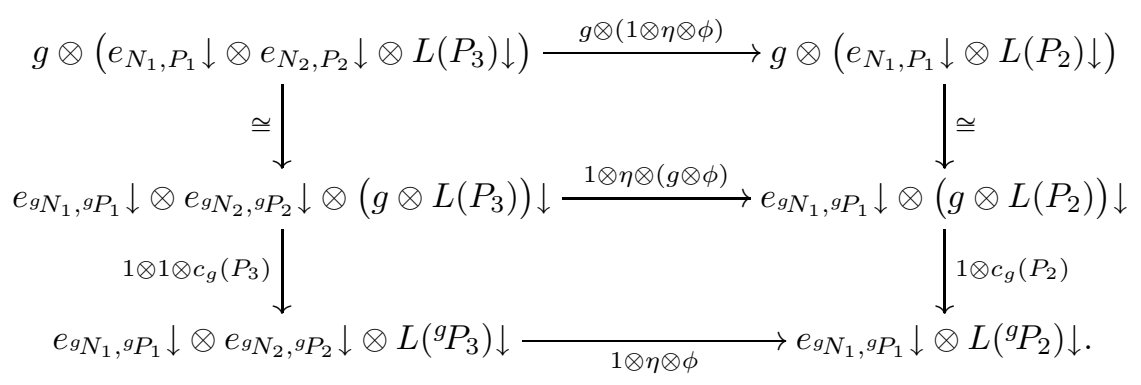

Combining this diagram with the analogous diagram in which $P_{1}$ and $P_{2}$ are interchanged, we conclude that the first diagram given in the statement of the lemma commutes.

Finally, if $P_{1} \subseteq P_{2}$ and $P \in \mathcal{P}(G)$, then it is straightforward to verify that the last diagram given in the statement of the lemma commutes.

Proposition 4.2. Let $L$ be a $G$-local module, and let $P \in \mathcal{P}(G)$. Then there is an isomorphism $\mathfrak{F}\left(L(P) \uparrow^{G}\right) \cong e_{N, P}^{\uparrow G} \otimes L$, and this isomorphism is natural in $L$.

Proof. Let $P_{0} \in \mathcal{P}(G)$. By Lemma 4.1 there are isomorphisms

$$
\begin{aligned}
e_{N_{0}, P_{0}} \otimes L(P) \uparrow^{G} \downarrow_{N_{0}} & \cong \bigoplus_{g \in N_{0} \backslash G / N} e_{N_{0}, P_{0}} \otimes(g \otimes L(P)) \downarrow_{g_{N} \cap N_{0}} \uparrow^{N_{0}} \\
& \cong \bigoplus_{g}\left(e_{N_{0}, P_{0} \downarrow_{g_{N} \cap N_{0}}} \otimes L\left({ }^{g} P\right) \downarrow_{g_{N} \cap N_{0}}\right) \uparrow^{N_{0}} \\
& \cong \bigoplus_{g}\left(e_{g_{N, g_{P}} \downarrow_{g_{N} \cap N_{0}}} \otimes L\left(P_{0}\right) \downarrow_{g_{N} \cap N_{0}}\right) \uparrow^{N_{0}} \\
& \cong \bigoplus_{g}\left(g \otimes e_{N, P}\right) \downarrow_{g_{N} \cap N_{0}} \uparrow^{N_{0}} \otimes L\left(P_{0}\right) \\
& \cong e_{N, P}^{\uparrow G} \downarrow_{N_{0}} \otimes L\left(P_{0}\right),
\end{aligned}
$$

and it is straightforward to check that these isomorphisms are natural in $L$. Let $\Phi\left(P_{0}\right): e_{N_{0}, P_{0}} \otimes L(P) \uparrow^{G} \downarrow_{N_{0}} \rightarrow e_{N, P}^{\uparrow G} \downarrow_{N_{0}} \otimes L\left(P_{0}\right)$ be the above isomorphism. Then it is only necessary to show that $\Phi: \mathfrak{F}\left(L(P) \uparrow^{G}\right) \rightarrow e_{N, P}^{\uparrow G} \otimes L$ is a $G$-local homomorphism.

Suppose that $P_{1}, P_{2} \in \mathcal{P}(G)$ with $P_{1} \subseteq P_{2}$. We wish to show that there is a commutative diagram

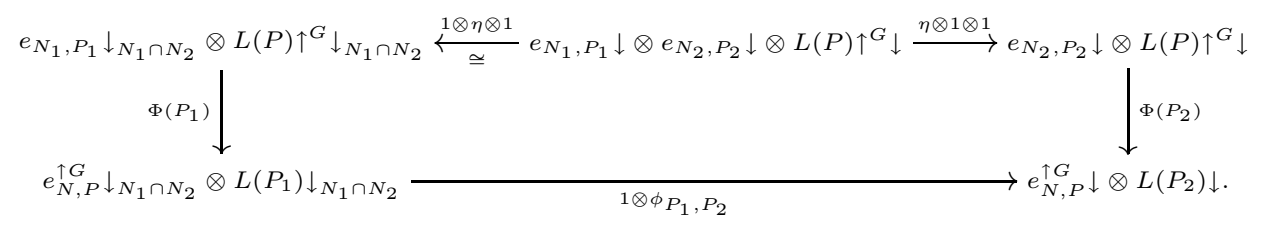


If $g \in G$, then Lemma4.1] shows that in $k\left[{ }^{g} N \cap N_{1} \cap N_{2}\right]$-Mod there is a commutative diagram given by

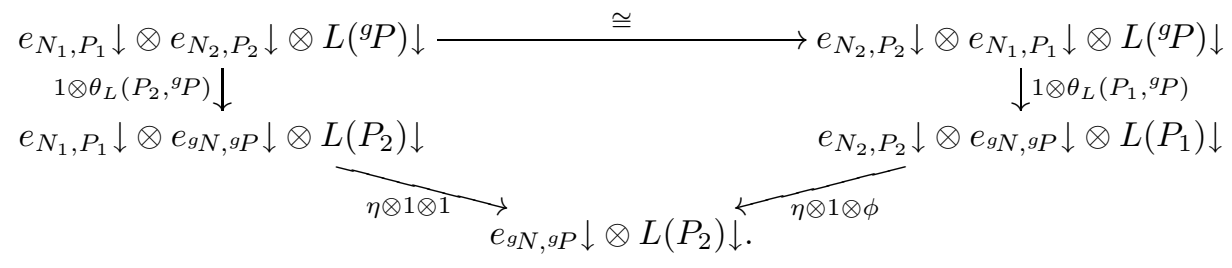

After inducing from ${ }^{g} N \cap N_{1} \cap N_{2}$ to $N_{1} \cap N_{2}$ and taking the direct sum over $g \in\left(N_{1} \cap N_{2}\right) \backslash G / N$, some tedious verifications show that there is a commutative diagram

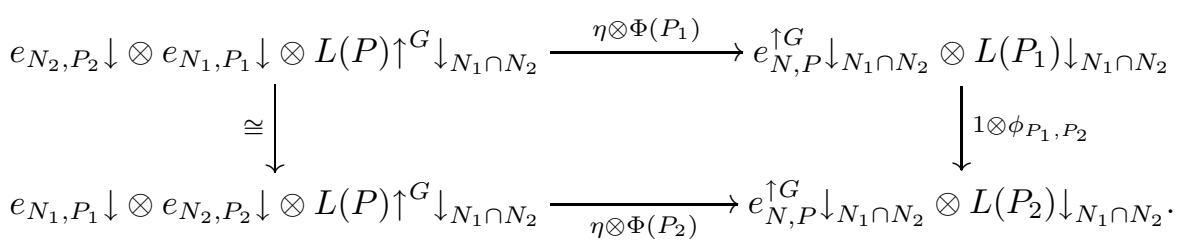

It is easy to see that this is equivalent to the commutativity of the desired diagram.

Finally, suppose that $g \in G$ and that $P_{0} \in \mathcal{P}(G)$. We must show that there is a commutative diagram in $k\left[{ }^{g} N_{0}\right]$-Mod of the form

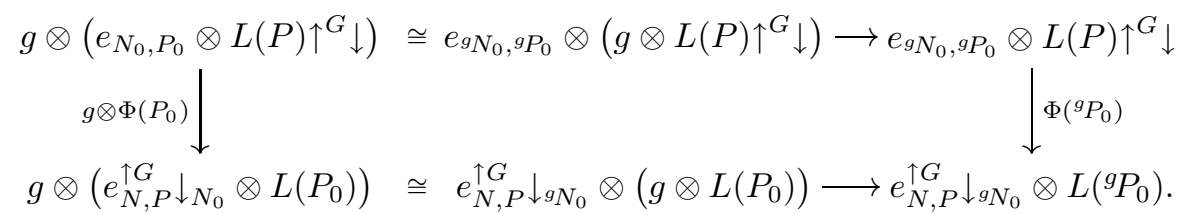

Let $T$ be a set of representatives for the $\left(N_{0}, N\right)$ double cosets in $G$. It follows from Lemma 4.1 that there is a commutative diagram

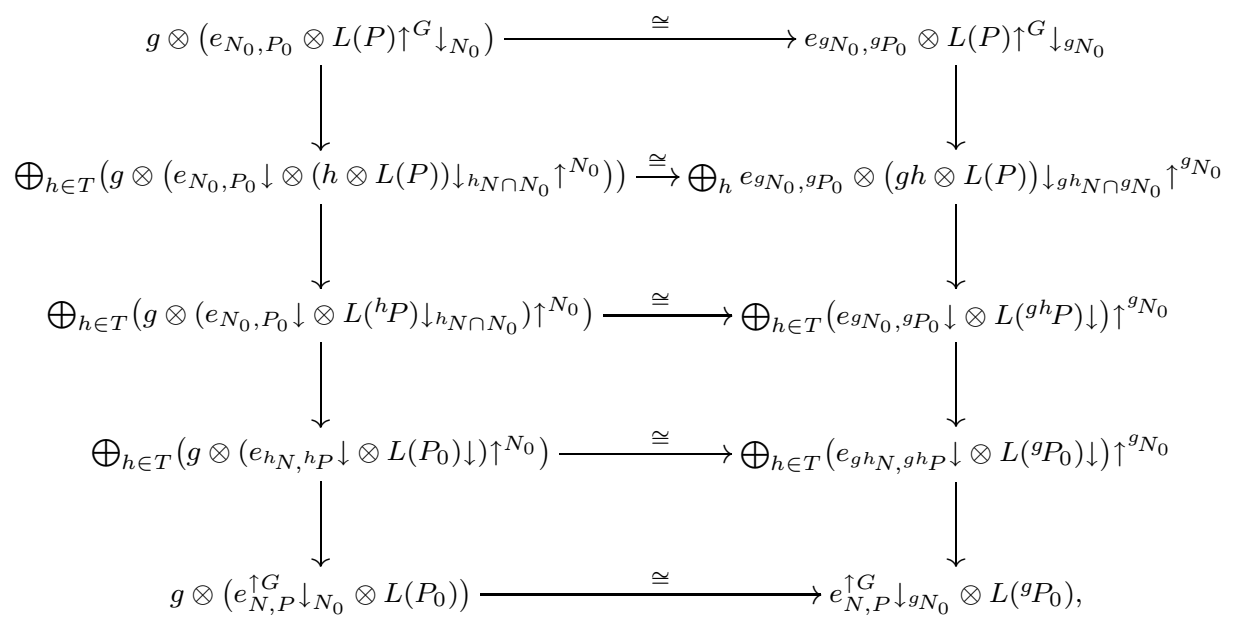

and this completes the proof.

It is possible to define not only the tensor product but also operations such as induction and restriction of $G$-local modules. In the case of restriction, for example, 
let $L$ be a $G$-local module, and let $H \subseteq G$. Define an $H$-local module $L \downarrow_{H}$ by setting

$$
L \downarrow_{H}(P)=L(P) \downarrow_{H \cap N}
$$

for every $p$-subgroup $P \subseteq H$. Note that $\bar{V}_{N}(L(P)) \subseteq V_{N, P}$, so

$$
\bar{V}_{H \cap N}\left(L \downarrow_{H}(P)\right)=\left(\operatorname{res}_{N, H \cap N}^{*}\right)^{-1}\left(\bar{V}_{N}(L(P))\right) \subseteq\left(\operatorname{res}_{N, H \cap N}^{*}\right)^{-1}\left(V_{N, P}\right)=V_{H \cap N, P} .
$$

If $P_{1}$ and $P_{2}$ are $p$-subgroups of $H$ with $P_{1} \subseteq P_{2}$, then the map $\phi_{P_{1}, P_{2}}: L\left(P_{1}\right) \downarrow_{N_{1} \cap N_{2}}$ $\rightarrow L\left(P_{2}\right) \downarrow_{N_{1} \cap N_{2}}$ gives a commutative diagram

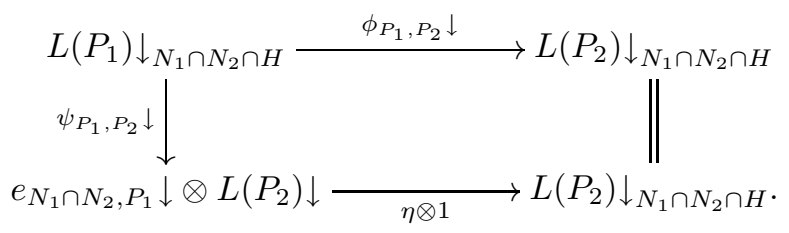

Moreover, $\left(\operatorname{res}_{N_{1} \cap N_{2}, N_{1} \cap N_{2} \cap H}^{*}\right)^{-1}\left(V_{N_{1} \cap N_{2}, P_{1}}\right)=V_{N_{1} \cap N_{2} \cap H, P_{1}}$ so that

$$
e_{N_{1} \cap N_{2}, P_{1}} \downarrow_{N_{1} \cap N_{2} \cap H} \cong e_{N_{1} \cap N_{2} \cap H, P_{1}}
$$

Finally, if $h \in H$, then $c_{h}(P): h \otimes L(P) \rightarrow L\left({ }^{h} P\right)$ is a stable isomorphism, so $c_{h}(P) \downarrow_{h_{N \cap H}}: h \otimes L \downarrow_{H}(P) \rightarrow L \downarrow_{H}\left({ }^{h} P\right)$ is also a stable isomorphism. It is easy to check that the resulting object $L \downarrow_{H}=\left(L \downarrow_{H}, \phi \downarrow_{H}, c \downarrow_{H}\right)$ is an $H$-local module. If $\xi: L \rightarrow L^{\prime}$ is a $G$-local homomorphism, set $\xi \downarrow_{H}(P)=\xi(P) \downarrow_{N \cap H}: L(P) \downarrow_{N \cap H} \rightarrow$ $L^{\prime}(P) \downarrow_{N \cap H}$ for every $p$-subgroup $P$ of $H$. Then $\xi \downarrow_{H}: L \downarrow_{H} \rightarrow L^{\prime} \downarrow_{H}$ is an $H$-local homomorphism, so there is a restriction functor $\operatorname{res}_{G, H}: \mathfrak{L}(G, k) \rightarrow \mathfrak{L}(H, k)$.

Using arguments similar to those given in the proof of Proposition 4.2 one can show that for any $P \in \mathcal{P}(G)$ there is a natural isomorphism $\mathfrak{F}(L(P)) \cong e_{N, P} \otimes L \downarrow_{N}$ in $\mathfrak{L}(N, k)$. It is much easier, however, to deduce the existence of this isomorphism from Theorem 5.9. so we postpone any further discussion of the restriction functor to the next section.

\section{An equivalence of CATEgories}

The current section is devoted to showing that the canonical functor $\mathfrak{F}: k G$-Mod $\rightarrow \mathfrak{L}(G, k)$ is an equivalence of categories. Constructing an explicit adjoint seems to be rather difficult, so we prove that $\mathfrak{F}$ is an equivalence by showing that it is essentially surjective, full, and faithful. As a first step toward proving faithfulness, we begin with a result showing that any $G$-local homomorphism is uniquely determined by its value on a Sylow $p$-subgroup.

Proposition 5.1. Let $L$ and $L^{\prime}$ be $G$-local modules, and let $\xi: L \rightarrow L^{\prime}$ be a $G$-local homomorphism such that $\xi(P)=0$ for some Sylow p-subgroup $P$ of $G$. Then $\xi=0$.

Proof. Let $Q$ be a $p$-subgroup of $G$, and let $R$ be a Sylow $p$-subgroup of $N_{G}(Q)$. Then there is an element $g \in G$ with ${ }^{g} R \subseteq P$. The commutative diagram

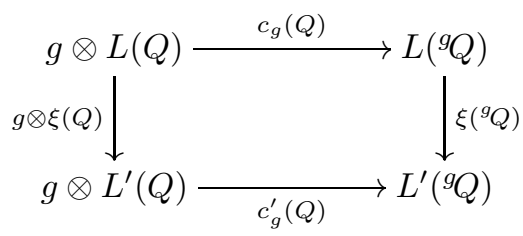

shows that $\xi(Q)=0$ if and only if $\xi\left({ }^{g} Q\right)=0$. In order to show that $\xi(Q)=0$, then, we may replace $Q$ by ${ }^{g} Q$ and may therefore assume that $g=1$ and that $R \subseteq P$. 
The commutative diagram

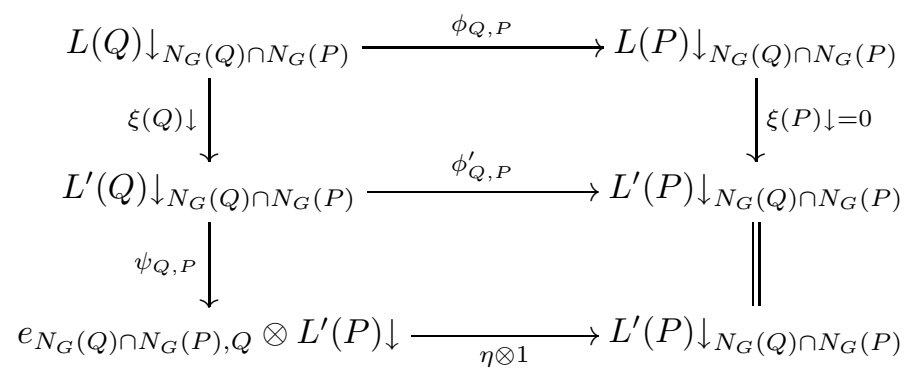

shows that $(\eta \otimes 1) \circ \psi_{Q, P} \circ \xi(Q) \downarrow=0$. But $\bar{V}_{N_{G}(Q) \cap N_{G}(P)}(L(Q) \downarrow) \subseteq V_{N_{G}(Q) \cap N_{G}(P), Q}$, so the map $(\eta \otimes 1) \circ \psi_{Q, P} \circ \xi(Q) \downarrow$ factors uniquely through $\eta \otimes 1$, and hence $\psi_{Q, P} \circ$ $\xi(Q) \downarrow=0$. Because $\psi_{Q, P}$ is a stable isomorphism, it follows that $\xi(Q) \downarrow_{N_{G}(Q) \cap N_{G}(P)}$ $=0$ and hence $\xi(Q) \downarrow_{R}=0$. Thus

$$
\xi(Q)=\frac{1}{\left|N_{G}(Q): R\right|} \operatorname{Tr}_{R}^{N_{G}(Q)}\left(\xi(Q) \downarrow_{R}\right)=0,
$$

as desired.

Let $P$ be a Sylow $p$-subgroup of $G$. Set $N=N_{G}(P)$, and let $g \in G$. If $Q$ is a Sylow $p$-subgroup of ${ }^{g} N \cap N$, then $Q \subseteq{ }^{g} P \cap P \subseteq{ }^{g} N \cap N$, and hence $Q={ }^{g} P \cap P$. It follows that the map $\eta: e_{g_{N} \cap N,{ }^{g} P \cap P} \rightarrow k$ is a stable isomorphism, and hence so are

$$
\phi_{g_{P} \cap P,{ }^{g} P}: L\left({ }^{g} P \cap P\right) \downarrow_{g_{N} \cap N} \rightarrow L\left({ }^{g} P\right) \downarrow_{g_{N} \cap N}
$$

and

$$
\phi_{g_{P} \cap P, P}: L\left({ }^{g} P \cap P\right) \downarrow_{g_{N} \cap N} \rightarrow L(P) \downarrow_{g_{N} \cap N} .
$$

Definition 5.2. Let $L$ and $L^{\prime}$ be $G$-local modules, let $P$ be a Sylow $p$-subgroup of $G$, and set $N=N_{G}(P)$. Suppose that $\gamma: L(P) \rightarrow L^{\prime}(P)$ is a $k N$-homomorphism. We say that $\gamma$ is $G$-stable if there is a commutative diagram in $k\left[{ }^{g} N \cap N\right]$-Mod of the form

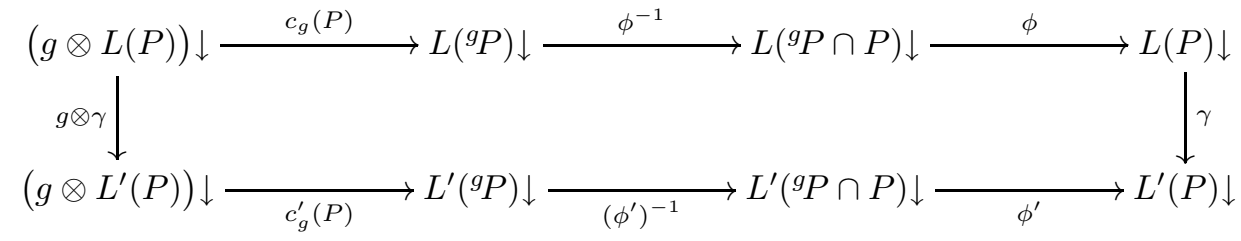

for all $g \in G$.

The following result is easy to verify, and the proof is left to the reader.

Proposition 5.3. Let $L$ and $L^{\prime}$ be $G$-local modules, let $P$ be a Sylow p-subgroup of $G$, and set $N=N_{G}(P)$. Suppose that $\gamma: L(P) \rightarrow L^{\prime}(P)$ is a $k N$-homomorphism. If there is a $G$-local homomorphism $\xi: L \rightarrow L^{\prime}$ with $\xi(P)=\gamma$, then $\gamma$ is $G$-stable.

Let $L$ and $L^{\prime}$ be $G$-local modules, and let $P$ be a Sylow $p$-subgroup of $G$. Set $N=N_{G}(P)$. We will define a homomorphism

$$
T=T_{P}^{L, L^{\prime}}: \underline{\operatorname{Hom}}_{k N}\left(L(P), L^{\prime}(P)\right) \rightarrow \operatorname{Hom}_{\mathfrak{L}(G, k)}\left(L, L^{\prime}\right) .
$$


Because $P$ is a Sylow $p$-subgroup of $G$, we have $e_{N, P} \cong k_{N}$. The maps $\iota: k_{G} \rightarrow k_{N}^{\uparrow G}$ and $\varepsilon: k_{N}^{\uparrow G} \rightarrow k_{G}$ given by $\iota(x)=\sum_{g \in G / N} g \otimes x$ and $\varepsilon\left(\sum_{g \in G / N} g \otimes x_{g}\right)=\sum_{g} x_{g}$ satisfy $\varepsilon \iota=1_{k}$ because $|G: N| \equiv 1(\bmod p)$. If $\gamma: L(P) \rightarrow L^{\prime}(P)$ is a $k N$ homomorphism, let $T \gamma: L \rightarrow L^{\prime}$ be the $G$-local homomorphism given by the composition

$$
\begin{aligned}
L \stackrel{\iota \otimes 1}{\longrightarrow} k_{N}^{\uparrow G} \otimes L & \cong e_{N, P}^{\uparrow G} \otimes L \cong \mathfrak{F}\left(L(P) \uparrow^{G}\right) \stackrel{\mathfrak{F}\left(\gamma \uparrow^{G}\right)}{\longrightarrow} \mathfrak{F}\left(L^{\prime}(P) \uparrow^{G}\right) \\
& \cong e_{N, P}^{\uparrow G} \otimes L^{\prime} \cong k_{N}^{\uparrow G} \otimes L^{\prime} \stackrel{\varepsilon \otimes 1}{\longrightarrow} L^{\prime} .
\end{aligned}
$$

Proposition 5.4. Let $L$ and $L^{\prime}$ be $G$-local modules, and let $P$ be a Sylow p-subgroup of $G$. Set $N=N_{G}(P)$, and let $\gamma: L(P) \rightarrow L^{\prime}(P)$ be a $k N$-homomorphism.

(1) If $\gamma$ is $G$-stable, then $T \gamma: L \rightarrow L^{\prime}$ is a $G$-local homomorphism with $(T \gamma)(P)=$ $\gamma$.

(2) If $L=\mathfrak{F} M$ and $L^{\prime}=\mathfrak{F} M^{\prime}$ for some $k G$-modules $M$ and $M^{\prime}$, then $T \gamma=$ $\mathfrak{F}\left(\operatorname{Tr}_{N}^{G} \gamma\right)$.

Proof. To compute $(T \gamma)(P)$, we begin by observing that the definition of the isomorphism in Proposition 4.2 shows that the composition of the isomorphisms

$$
\begin{aligned}
L(P) \uparrow^{G} \downarrow_{N} & \cong e_{N, P} \otimes L(P) \uparrow^{G} \downarrow_{N}=\mathfrak{F}\left(L(P) \uparrow^{G}\right)(P) \\
& \cong e_{N, P}^{\uparrow G} \downarrow_{N} \otimes L(P) \cong k_{N}^{\uparrow G} \downarrow_{N} \otimes L(P)
\end{aligned}
$$

is given by

$$
\begin{aligned}
L(P) \uparrow^{G} \downarrow_{N} & \cong \bigoplus_{g \in N \backslash G / N}(g \otimes L(P)) \downarrow_{g_{N} \cap N} \uparrow^{N} \\
& \cong \bigoplus_{g} L\left({ }^{g} P\right) \downarrow_{g_{N \cap N} \uparrow^{N}} \\
& \cong \bigoplus_{g} L\left({ }^{g} P \cap P\right) \downarrow_{g_{N} \cap N} \uparrow^{N} \\
& \cong \bigoplus_{g} L(P) \downarrow_{g_{N \cap N} \uparrow^{N}} \\
& \cong \bigoplus_{g} k_{g_{N} \uparrow N}^{\uparrow G} \otimes L(P) \\
& \cong k_{N}^{\uparrow G} \downarrow_{N} \otimes L(P) .
\end{aligned}
$$

If $\gamma$ is $G$-stable, then there is a commutative diagram

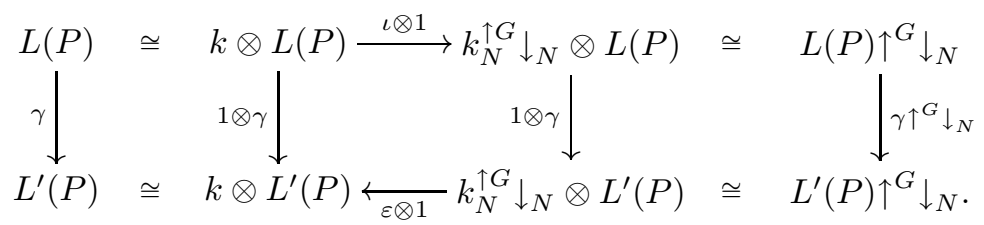

Hence $(T \gamma)(P)=\gamma$, and (1) holds.

Now suppose that $L=\mathfrak{F} M$ and $L^{\prime}=\mathfrak{F} M^{\prime}$. Identifying $e_{N, P}$ with $k_{N}$, we may assume that $L(P)=M \downarrow_{N}$ and $L^{\prime}(P)=M^{\prime} \downarrow_{N}$. Then the isomorphism

$$
k_{N}^{\uparrow G} \downarrow_{N} \otimes L(P)=k_{N}^{\uparrow G} \downarrow_{N} \otimes M \downarrow_{N} \cong M \downarrow_{N} \uparrow^{G} \downarrow_{N}=L(P) \uparrow^{G} \downarrow_{N}
$$


is given by $(g \otimes 1) \otimes m \mapsto g \otimes g^{-1} m$, and similarly for $L^{\prime}$ instead of $L$. It follows that the composition

$$
\begin{aligned}
M \downarrow_{N} & \stackrel{\iota \otimes 1}{\longrightarrow} k_{N}^{\uparrow G} \downarrow_{N} \otimes M \downarrow_{N} \\
\quad \stackrel{\gamma \uparrow^{G} \downarrow_{N}}{\longrightarrow} M^{\prime} \downarrow_{N} \uparrow^{G} \downarrow_{N} & \cong \downarrow_{N} \uparrow_{N}^{\uparrow G} \downarrow_{N} \otimes \downarrow_{N} \\
& M^{\prime} \downarrow_{N} \stackrel{\varepsilon \otimes 1}{\longrightarrow} M^{\prime} \downarrow_{N}
\end{aligned}
$$

is equal to $\operatorname{Tr}_{N}^{G} \gamma$. Thus $T \gamma: \mathfrak{F} M \rightarrow \mathfrak{F} M^{\prime}$ and $\mathfrak{F}\left(\operatorname{Tr}_{N}^{G} \gamma\right): \mathfrak{F} M \rightarrow \mathfrak{F} M^{\prime}$ are $G$-local homomorphisms with $(T \gamma)(P)=\mathfrak{F}\left(\operatorname{Tr}_{N}^{G} \gamma\right)(P)$. Proposition 5.1 now implies that $T \gamma=\mathfrak{F}\left(\operatorname{Tr}_{N}^{G} \gamma\right)$, as desired.

Corollary 5.5. The functor $\mathfrak{F}: k G-\underline{\operatorname{Mod}} \rightarrow \mathfrak{L}(G, k)$ is full.

Proof. Let $M$ and $M^{\prime}$ be $k G$-modules, and let $\xi: \mathfrak{F} M \rightarrow \mathfrak{F} M^{\prime}$ be a $G$-local homomorphism. Let $P$ be a Sylow $p$-subgroup of $G$, and set $N=N_{G}(P)$. Then $\xi(P)$ is $G$-stable, so $T \xi(P): \mathfrak{F} M \rightarrow \mathfrak{F} M^{\prime}$ satisfies $(T \xi(P))(P)=\xi(P)$. Hence $\xi=T \xi(P)=\mathfrak{F}\left(\operatorname{Tr}_{N}^{G} \xi(P)\right)$, and $\mathfrak{F}$ is full.

As another consequence of Proposition 5.4, we present a result that strengthens the statement of Proposition 3.1

Corollary 5.6. Let $\xi: L \rightarrow L^{\prime}$ be a G-local homomorphism, and let $P$ be a Sylow p-subgroup of $G$. Then $\xi$ is an isomorphism if and only if $\xi(P)$ is an isomorphism.

Proof. It is clear that if $\xi$ is an isomorphism, then $\xi(P)$ is an isomorphism. To prove the converse, suppose that $\xi(P)$ is an isomorphism. Because $\xi(P)$ is $G$-stable, the map $\xi(P)^{-1}: L^{\prime}(P) \rightarrow L(P)$ is also $G$-stable. By Proposition 5.4 there is a $G$-local homomorphism $\xi^{\prime}: L^{\prime} \rightarrow L$ with $\xi^{\prime}(P)=\xi(P)^{-1}$. Then $\left(\xi^{\prime} \xi\right)(P)=1_{L(P)}$ and $\left(\xi \xi^{\prime}\right)(P)=1_{L^{\prime}(P)}$, so $\xi^{\prime} \xi=1_{L}$ and $\xi \xi^{\prime}=1_{L^{\prime}}$ by Proposition 5.1 Thus $\xi$ is an isomorphism.

The following technical lemma is needed to show that the functor $\mathfrak{F}$ is essentially surjective.

Lemma 5.7. Let $\xi_{1}: L_{1} \rightarrow L_{1}^{\prime}$ and $\xi_{2}: L_{2} \rightarrow L_{2}^{\prime}$ be G-local homomorphisms, and let $P$ be a Sylow p-subgroup of $G$. Set $N=N_{G}(P)$. Suppose that $\gamma: L_{1}(P) \rightarrow$ $L_{2}(P)$ and $\gamma^{\prime}: L_{1}^{\prime}(P) \rightarrow L_{2}^{\prime}(P)$ are $k N$-homomorphisms such that there is a commutative diagram

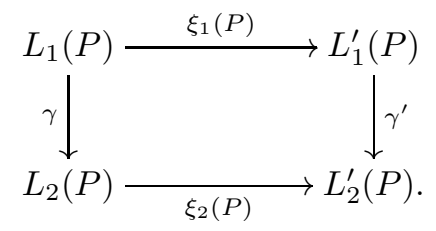

Then $\left(T \gamma^{\prime}\right) \circ \xi_{1}=\xi_{2} \circ(T \gamma)$.

Proof. The result follows immediately from the commutativity of the diagram

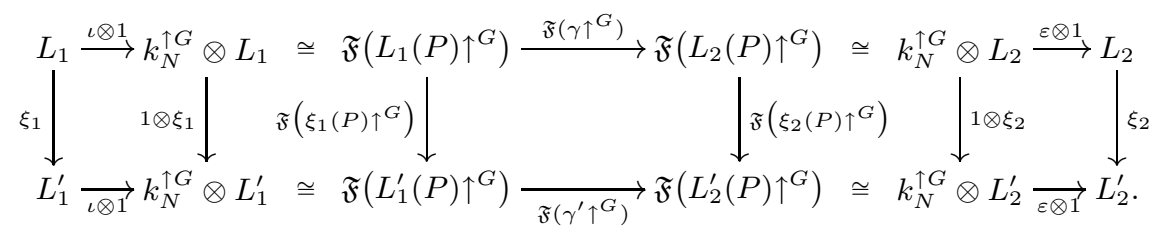


Let $r$ denote the $p$-rank of $G$, and suppose that $s$ is an integer with $0 \leq s \leq r$. Let $k G$-Mod $(s)$ denote the full subcategory of $k G$-Mod consisting of all modules $M$ with $e_{s} \otimes M \cong M$, and let $\mathfrak{L}_{s}(G, k)$ be the full subcategory of $\mathfrak{L}(G, k)$ consisting of all objects $L$ such that $e_{s} \otimes L \cong L$. It is easy to check that if $M$ is any $k G$ module, then $M$ is in $k G-\operatorname{Mod}(s)$ if and only if $\mathfrak{F} M$ is in $\mathfrak{L}_{s}(G, k)$. In particular, the restriction of $\mathfrak{F}$ to $k G-\underline{\operatorname{Mod}}(s)$ defines a functor $\mathfrak{F}_{s}: k G$ - $\underline{\operatorname{Mod}}(s) \rightarrow \mathfrak{L}_{s}(G, k)$.

Lemma 5.8. Let $0 \leq s \leq r-1$, and assume that the functor $\mathfrak{F}_{s}: k G-\underline{\operatorname{Mod}}(s) \rightarrow$ $\mathfrak{L}_{s}(G, k)$ is essentially surjective. Then $\mathfrak{F}_{s+1}$ is also essentially surjective.

Proof. Let $L$ be an object of $\mathfrak{L}_{s+1}(G, k)$. Then $e_{s} \otimes L$ is an object of $\mathfrak{L}_{s}(G, k)$, so there is a module $M$ in $k G-\underline{\operatorname{Mod}}(s)$ such that $\mathfrak{F} M \cong e_{s} \otimes L$. Let $\xi: \mathfrak{F} M \rightarrow L$ denote the composition

$$
\mathfrak{F} M \cong e_{s} \otimes L \stackrel{\eta \otimes 1}{\longrightarrow} L .
$$

Then $1 \otimes \xi: e_{s} \otimes \mathfrak{F} M \rightarrow e_{s} \otimes L$ is an isomorphism.

Let $E_{1}, \ldots, E_{n}$ be representatives for the conjugacy classes of elementary abelian $p$-subgroups of rank $s+1$ in $G$. Fix $i$ with $1 \leq i \leq n$, and consider the map $\beta_{E_{i}}=\beta_{s} \otimes 1$ occurring in the triangle

$$
e_{s} \downarrow_{N_{i}} \otimes L\left(E_{i}\right) \longrightarrow L\left(E_{i}\right) \longrightarrow f_{s} \downarrow_{N_{i}} \otimes L\left(E_{i}\right) \stackrel{\beta_{s} \otimes 1}{\longrightarrow} \Omega^{-1} k \otimes e_{s} \downarrow_{N_{i}} \otimes L\left(E_{i}\right) .
$$

Using the isomorphisms

$$
\begin{aligned}
& \underline{\operatorname{Hom}}_{k N_{i}}\left(f_{s} \downarrow_{N_{i}} \otimes L\left(E_{i}\right), \Omega^{-1} k \otimes e_{s} \downarrow_{N_{i}} \otimes L\left(E_{i}\right)\right) \\
& \quad \cong \underline{\operatorname{Hom}}_{k N_{i}}\left(f_{s} \downarrow_{N_{i}} \otimes L\left(E_{i}\right), \Omega^{-1} k \otimes(\mathfrak{F} M)\left(E_{i}\right)\right) \\
& \quad=\underline{\operatorname{Hom}}_{k N_{i}}\left(f_{s} \downarrow \otimes L\left(E_{i}\right), \Omega^{-1} k \otimes e_{N_{i}, E_{i}} \otimes M \downarrow\right) \\
& \quad \cong \underline{\operatorname{Hom}}_{k N_{i}}\left(f_{s} \downarrow_{N_{i}} \otimes L\left(E_{i}\right), \Omega^{-1} k \otimes M \downarrow_{N_{i}}\right) \\
& \quad \cong \underline{\operatorname{Hom}}_{k G}\left(f_{s} \otimes L\left(E_{i}\right) \uparrow^{G}, \Omega^{-1} k \otimes M\right),
\end{aligned}
$$

we obtain a map $\beta_{E_{i}}^{\prime}: f_{s} \otimes L\left(E_{i}\right)^{G} \rightarrow \Omega^{-1} k \otimes M$ corresponding to $\beta_{E_{i}}$. Set $M_{s+1}=\bigoplus_{i=1}^{n} L\left(E_{i}\right) \uparrow^{G}$. Taking the direct sum of the maps $\beta_{E_{i}}^{\prime}$ for $1 \leq i \leq n$ gives a map $\beta: f_{s} \otimes M_{s+1} \rightarrow \Omega^{-1} k \otimes M$. Let $M^{\prime}$ be the $k G$-module defined by the triangle

$$
M \stackrel{\mu}{\longrightarrow} M^{\prime} \longrightarrow f_{s} \otimes M_{s+1} \stackrel{\beta}{\longrightarrow} \Omega^{-1} k \otimes M .
$$

Then $\bar{V}_{G}\left(M^{\prime}\right) \subseteq \bar{V}_{G}(M) \cup \bar{V}_{G}\left(f_{s} \otimes M_{s+1}\right) \subseteq V_{s+1}$, so $M^{\prime}$ is an object of $k G$ $\underline{\operatorname{Mod}}(s+1)$. We will show that $\mathfrak{F} M^{\prime} \cong L$.

Observe that for $1 \leq i \leq n$ the map $\beta_{E_{i}}^{\prime}: f_{s} \otimes L\left(E_{i}\right) \uparrow^{G} \rightarrow \Omega^{-1} k \otimes M$ factors as

$$
\begin{aligned}
& f_{s} \otimes L\left(E_{i}\right) \uparrow^{G} \stackrel{\beta_{s} \otimes 1}{\longrightarrow} \Omega^{-1} k \otimes e_{s} \otimes L\left(E_{i}\right) \uparrow^{G} \cong \Omega^{-1} k \otimes\left(e_{s} \downarrow_{N_{i}} \otimes L\left(E_{i}\right) \uparrow^{G}\right. \\
& \cong \Omega^{-1} k \otimes\left(e_{N_{i}, E_{i}} \otimes M \downarrow_{N_{i}}\right) \uparrow^{G} \cong \Omega^{-1} k \otimes e_{N_{i}, E_{i}}^{\uparrow G} \otimes M \stackrel{1 \otimes \varepsilon \eta_{i} \uparrow^{G} \otimes 1}{\longrightarrow} \Omega^{-1} k \otimes M .
\end{aligned}
$$

Let $P$ be a Sylow $p$-subgroup of $G$, and set $N=N_{G}(P)$. Then the map $\left(\mathfrak{F} \beta_{E_{i}}^{\prime}\right)(P)$ in $k N$-Mod is given by the composition

$$
\begin{aligned}
e_{N, P} \otimes f_{s} \downarrow_{N} \otimes L\left(E_{i}\right) \uparrow^{G} \downarrow_{N} & \rightarrow e_{N, P} \otimes \Omega^{-1} k \otimes e_{N_{i}, E_{i}}^{\uparrow G} \otimes M \downarrow \\
& \stackrel{1 \otimes 1 \otimes \varepsilon \eta_{i} \uparrow^{G} \otimes 1}{\longrightarrow} e_{N, P} \otimes \Omega^{-1} k \otimes M \downarrow_{N} .
\end{aligned}
$$


After a permutation of the tensor factors, the first map in this composition is just the composition around the diagram

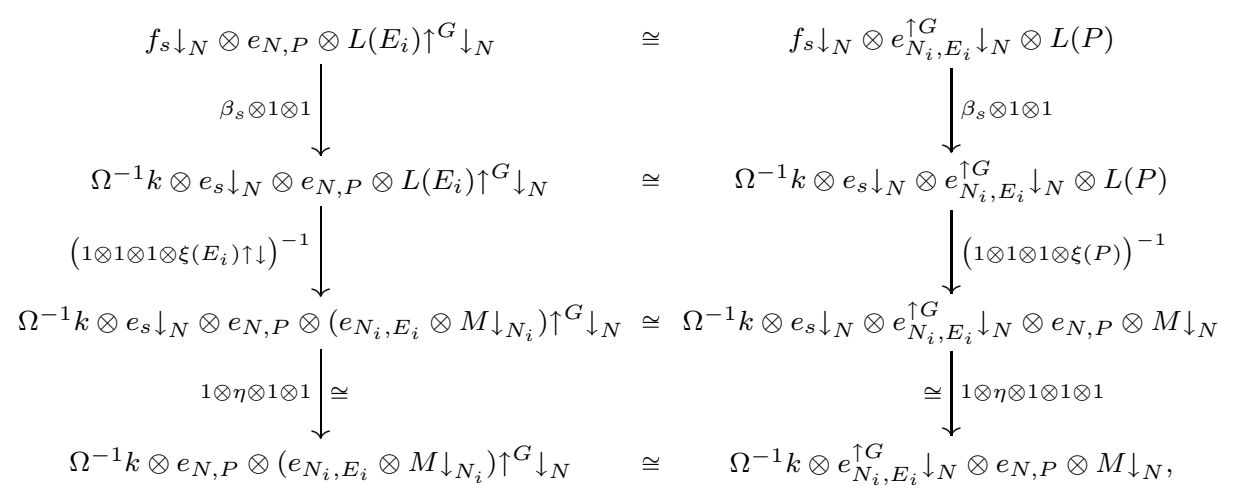

in which the middle square commutes by Proposition 4.2 It follows, therefore, that the triangle in which the third map is

$$
e_{N, P} \otimes f_{s} \downarrow_{N} \otimes M_{s+1} \downarrow_{N} \stackrel{1 \otimes \beta}{\longrightarrow} e_{N, P} \otimes \Omega^{-1} k \otimes M \downarrow_{N} \cong \Omega^{-1} k \otimes e_{N, P} \otimes M \downarrow_{N}
$$

is isomorphic to the triangle in which the third map is given by the composition

$$
\begin{aligned}
f_{s} \downarrow_{N} & \otimes\left(\bigoplus_{i=1}^{n} e_{N_{i}, E_{i}}^{\uparrow G} \downarrow_{N}\right) \otimes L(P) \stackrel{\beta_{s} \otimes 1 \otimes 1}{\longrightarrow} \Omega^{-1} k \otimes e_{s} \downarrow_{N} \otimes\left(\bigoplus_{i=1}^{n} e_{N_{i}, E_{i}}^{\uparrow G} \downarrow_{N}\right) \otimes L(P) \\
& \stackrel{(1 \otimes 1 \otimes 1 \otimes \xi(P))^{-1}}{\longrightarrow} \Omega^{-1} k \otimes e_{s} \downarrow_{N} \otimes\left(\bigoplus_{i=1}^{n} e_{N_{i}, E_{i}}^{\uparrow G} \downarrow_{N}\right) \otimes e_{N, P} \otimes M \downarrow_{N} \\
& \stackrel{1 \otimes 1 \otimes\left(\oplus \varepsilon \eta_{i} \uparrow \downarrow\right) \otimes 1 \otimes 1}{\longrightarrow} \Omega^{-1} k \otimes e_{s} \downarrow_{N} \otimes e_{N, P} \otimes M \downarrow_{N} .
\end{aligned}
$$

Let us call this composition $\tilde{\beta}$. For $1 \leq i \leq n$ let $\alpha_{i}: e_{N_{i}, E_{i}}^{\uparrow G} \rightarrow e_{s+1}$ be the map defined in Proposition 2.7, and let $\alpha: \bigoplus_{i=1}^{n} e_{N_{i}, E_{i}}^{\uparrow G} \rightarrow e_{s+1}$ be the direct sum of the $\alpha_{i}$. Then $\eta \alpha=\bigoplus_{i=1}^{n} \varepsilon \eta_{i} \uparrow^{G}$, and one can check that there is a commutative diagram

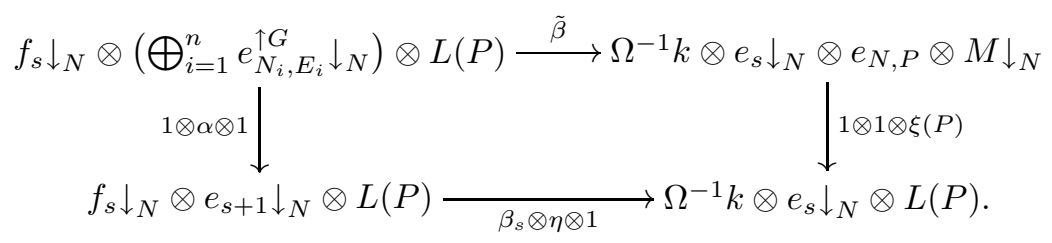

Now $1 \otimes \alpha: f_{s} \otimes\left(\bigoplus_{i=1}^{n} e_{N_{i}, E_{i}}^{\uparrow G}\right) \rightarrow f_{s} \otimes e_{s+1}$ is a stable isomorphism by Proposition [2.7, and $1 \otimes \xi(P): e_{s} \downarrow_{N} \otimes e_{N, P} \otimes M \downarrow_{N} \rightarrow e_{s} \downarrow_{N} \otimes L(P)$ is also an isomorphism. 
It follows that there are isomorphisms of triangles

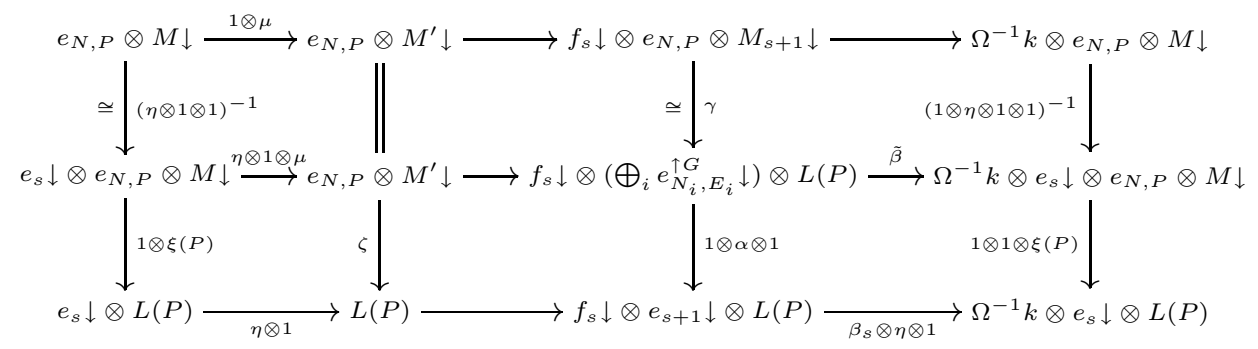

for some $\zeta: e_{N, P} \otimes M^{\prime} \downarrow_{N} \rightarrow L(P)$.

The first and last triangles in this commutative diagram correspond to $G$-local homomorphisms

$$
\mathfrak{F} M \stackrel{\mathfrak{F} \mu}{\longrightarrow} \mathfrak{F} M^{\prime} \longrightarrow f_{s} \otimes \mathfrak{F} M_{s+1} \longrightarrow \Omega^{-1} k \otimes \mathfrak{F} M
$$

and

$$
e_{s} \otimes L \stackrel{\eta \otimes 1}{\longrightarrow} L \longrightarrow f_{s} \otimes e_{s+1} \otimes L \longrightarrow \Omega^{-1} k \otimes e_{s} \otimes L .
$$

Because there are $G$-local homomorphisms

$$
\mathfrak{F} M \stackrel{(\eta \otimes 1)^{-1}}{\longrightarrow} e_{s} \otimes \mathfrak{F} M \stackrel{1 \otimes \xi}{\longrightarrow} e_{s} \otimes L
$$

and

$$
\begin{aligned}
f_{s} \otimes \mathfrak{F} M_{s+1} & \cong f_{s} \otimes \bigoplus_{i=1}^{n} \mathfrak{F}\left(L\left(E_{i}\right) \uparrow^{G}\right) \cong f_{s} \otimes\left(\bigoplus_{i=1}^{n} e_{N_{i}, E_{i}}^{\uparrow G}\right) \otimes L \\
& \stackrel{1 \otimes \alpha \otimes 1}{\longrightarrow} f_{s} \otimes e_{s+1} \otimes L,
\end{aligned}
$$

Proposition 5.3 shows that the compositions

$$
e_{N, P} \otimes M \downarrow_{N} \stackrel{(\eta \otimes 1 \otimes 1)^{-1}}{\longrightarrow} e_{s} \downarrow_{N} \otimes e_{N, P} \otimes M \downarrow_{N} \stackrel{1 \otimes \xi(P)}{\longrightarrow} e_{s} \downarrow_{N} \otimes L(P)
$$

and

$$
\begin{aligned}
f_{s} \downarrow_{N} \otimes e_{N, P} \otimes M_{s+1} \downarrow_{N} & \stackrel{\gamma}{\cong} f_{s} \downarrow \otimes\left(\bigoplus_{i=1}^{n} e_{N_{i}, E_{i}}^{\uparrow G} \downarrow\right) \otimes L(P) \\
& \stackrel{1 \otimes \alpha \otimes 1}{\longrightarrow} f_{s} \downarrow_{N} \otimes e_{s+1} \downarrow_{N} \otimes L(P)
\end{aligned}
$$

are both $G$-stable. Define

$$
\sigma=(1 \otimes \xi(P)) \circ(\eta \otimes 1 \otimes 1)^{-1}: e_{N, P} \otimes M \downarrow_{N} \rightarrow e_{s} \downarrow_{N} \otimes L(P) .
$$

Then Proposition 5.4 and Lemma 5.7 imply that there is a commutative diagram of triangles

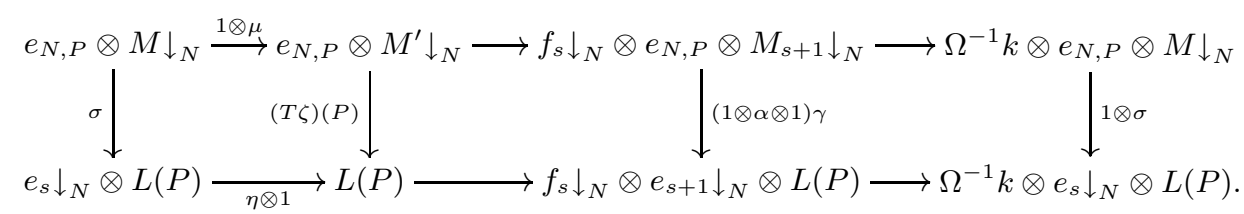

Hence $(T \zeta)(P): e_{N, P} \otimes M^{\prime} \downarrow_{N} \rightarrow L(P)$ is an isomorphism. Corollary 5.6 implies that $T \zeta: \mathfrak{F} M^{\prime} \rightarrow L$ is an isomorphism in $\mathfrak{L}_{s+1}(G, k)$, and this completes the proof. 
We can now prove the main result of the paper.

Theorem 5.9. The functor $\mathfrak{F}: k G-\underline{\operatorname{Mod}} \rightarrow \mathfrak{L}(G, k)$ is an equivalence of categories.

Proof. The functor $\mathfrak{F}_{0}: k G$ - $\underline{\operatorname{Mod}}(0) \rightarrow \mathfrak{L}_{0}(G, k)$ is trivially an equivalence because all objects of both $k G$ - $\underline{\operatorname{Mod}}(0)$ and $\mathfrak{L}_{0}(G, k)$ are isomorphic to zero. For $1 \leq s \leq r$ Lemma 5.8 implies by induction that $\mathfrak{F}_{s}$ is essentially surjective. In particular, $\mathfrak{F}=\mathfrak{F}_{r}$ is essentially surjective.

Now suppose that $\gamma: M \rightarrow M^{\prime}$ is a $k G$-homomorphism such that $\mathfrak{F} \gamma=0$. Let $P$ be a Sylow $p$-subgroup of $G$, and set $N=N_{G}(P)$. Identifying $e_{N, P}$ with $k_{N}$, we see that $0=(\mathfrak{F} \gamma)(P)=\gamma \downarrow_{N}$ and hence $\gamma=0$. Thus $\mathfrak{F}$ is faithful. Since $\mathfrak{F}$ is full by Corollary 5.5, it follows that $\mathfrak{F}$ is an equivalence, as desired.

Assume that $P$ is a Sylow $p$-subgroup of $G$, and set $N=N_{G}(P)$. Let $\mathfrak{l}(G, k)$ be the full subcategory of $\mathfrak{L}(G, k)$ consisting of the objects $L$ such that $L(P)$ is stably isomorphic to a finitely generated $k N$-module. If $M$ is a finitely generated $k G$-module, then $\mathfrak{F} M$ is an object of $\mathfrak{l}(G, k)$, so the restriction of $\mathfrak{F}$ defines a functor $\mathfrak{f}: k G$ - $\underline{\bmod } \rightarrow \mathfrak{l}(G, k)$.

Corollary 5.10. The functor $\mathfrak{f}: k G-\underline{\bmod } \rightarrow \mathfrak{l}(G, k)$ is an equivalence of categories.

Proof. Theorem 5.9 implies that $\mathfrak{f}$ is full and faithful, so it is only necessary to show that $\mathfrak{f}$ is essentially surjective. Let $L$ be an object of $\mathfrak{l}(G, k)$. Then there is a $k G$-module $M$ with $\mathfrak{F} M \cong L$ in $\mathfrak{L}(G, k)$, and we may assume without loss of generality that $M$ has no projective summands. The definition of $\mathfrak{l}(G, k)$ shows that $M \downarrow_{N} \cong M_{0} \oplus Q$ in $k N$-Mod for some finitely generated module $M_{0}$ and some projective module $Q$. But $M$ is a summand of $M \downarrow_{N} \uparrow^{G} \cong M_{0} \uparrow^{G} \oplus Q \uparrow^{G}$, and $M$ has no projective summands. Thus $M$ is a summand of $M_{0} \uparrow^{G}$, so that $M$ is finitely generated. It follows that $\mathfrak{f} M \cong L$ in $\mathfrak{l}(G, k)$, as desired.

We now prove the result on restrictions of $G$-local modules stated in Section 4 .

Corollary 5.11. Let $L$ be an object of $\mathfrak{L}(G, k)$, and let $P \in \mathcal{P}(G)$. Then there is an isomorphism $\mathfrak{F}(L(P)) \cong e_{N, P} \otimes L \downarrow_{N}$ in $\mathfrak{L}(N, k)$, and the isomorphism is natural in $L$.

Proof. Let $M$ be a $k G$-module such that there is an isomorphism $\xi: L \rightarrow \mathfrak{F} M$ in $\mathfrak{L}(G, k)$. Then we obtain isomorphisms

$$
\begin{aligned}
\mathfrak{F}(L(P)) \stackrel{\mathfrak{F}(\xi(P))}{\longrightarrow} \mathfrak{F}\left(e_{N, P} \otimes M \downarrow_{N}\right) & \cong e_{N, P} \otimes \mathfrak{F}\left(M \downarrow_{N}\right) \\
& \cong e_{N, P} \otimes(\mathfrak{F} M) \downarrow_{N} \cong e_{N, P} \otimes L \downarrow_{N}
\end{aligned}
$$

in $\mathfrak{L}(N, k)$. It is easy to check that the resulting isomorphism $\mathfrak{F}(L(P)) \cong e_{N, P} \otimes L \downarrow_{N}$ is natural.

If $B$ is a block of $k G$, then it seems reasonable to expect that there is a category that is equivalent to the stable category $B$-Mod and has a definition similar to that of $\mathfrak{L}(G, k)$. Unfortunately, the definition of $\mathfrak{L}(G, k)$ does not seem to be compatible with the block structure of $k G$. It is not clear, therefore, whether an appropriate construction can be obtained simply by modifying the techniques used here; significantly different ideas may be necessary. 


\section{REFERENCES}

[1] D. J. Benson, J. F. Carlson, and J. Rickard, Complexity and varieties for infinitely generated modules, II, Math. Proc. Cambridge Philos. Soc. 120 (1996), 597-615. MR 97f:20008

[2] D. Happel, Triangulated Categories in the Representation Theory of Finite Dimensional Algebras, Cambridge Univ. Press, Cambridge, 1988. MR 89e:16035

[3] J. Rickard, Idempotent modules in the stable category, J. London Math. Soc. (2) 56 (1997), 149-170. MR 998d:20058

[4] W. W. Wheeler, Quillen stratification for the stable module category, Quart. J. Math. Oxford (2) 50 (1999), 355-369. MR 2000m:20085

Center for Communications Research, 4320 Westerra Court, San Diego, California 92121

E-mail address: wheeler@member.ams.org 